\title{
Biosynthesized fluorinated organoborons
}

ACS Cent. Sci. https://go.nature.com/2Smd3ek (2019).

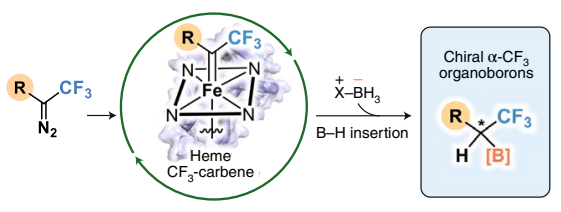

Credit: American Chemical Society, 2019

Trifluoromethylated organoborons are valuable synthetic building blocks as they provide access to a wide range of $\mathrm{CF}_{3}-$ containing molecules via boron-mediated transformations. However, their asymmetric synthesis remains a challenge.

Frances H. Arnold's group has pioneered the application of haem-containing enzymes for the catalysis of abiological carbene transfer reactions. Just one of the many impressive examples is the biocatalytic insertion of carbene reagents into $\mathrm{B}-\mathrm{H}$ bonds to give chiral organoborane compounds - achieved by reprogramming the enzyme Rhodothermus marinus cytochrome $c$.

Now, Kendall N. Houk, Frances H. Arnold and co-workers report the expansion of the synthetic scope of this enzyme to accept trifluorodiazo alkanes in asymmetric carbene $\mathrm{B}-\mathrm{H}$ bond insertion reactions for the synthesis of organofluorine compounds. For this purpose, directed evolution of the wild-type enzyme - that showed no considerable activity for the fluorinated substrates - was performed. Targeted residues for mutation were chosen in order to generate an enzyme active site that is surrounded by a more flexible loop, promoting substrate interactions with the carbene intermediate for increased enzyme activity, and also restricting the

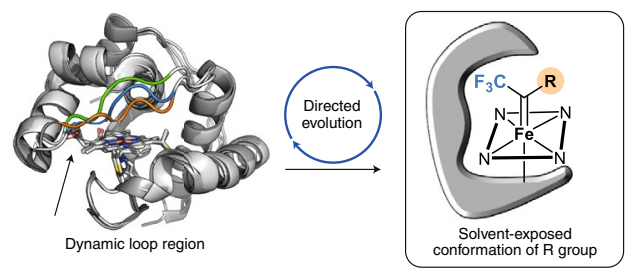

position and orientation of the carbene intermediate, whereby the $\mathrm{CF}_{3}$-group points towards the haem pocket and the alkyl substituent towards the solvent, for highly enantioselective $\mathrm{C}-\mathrm{B}$ bond formation and a broad substrate scope.

Indeed, several steps of directed evolution afforded a mutant enzyme - designated as BOR- $\mathrm{CF}_{3}$ - which allowed the conversion of a variety of structurally diverse, fluorinated diazoalkane substrates with good turnovers and high enantioselectivities. Notably, several of the synthesized chiral $\alpha-\mathrm{CF}_{3}$ organoborons appear to not be accessible by current alternative methods. Molecular dynamics simulations elucidated in detail the contributions of the mutations on the activity and stereospecificity of BOR-CF . $_{3}$.

Overall, this work is a valuable extension of an enzyme-catalysed abiological reaction, providing new opportunities for the synthetic chemist. The careful design of the study - taking structural and dynamical considerations into account - was the key to generating a broadly applicable and enantioselective biocatalyst.

Jan-Stefan Völler

Published online: 13 March 2019

https://doi.org/10.1038/s41929-019-0260-4 